\title{
Analyzing Planning Techniques for Whole Brain Radiotherapy
}

\author{
Soai Dang Quoc ${ }^{1}$, Quang Bui Vinh ${ }^{2}$, Cuong Bui Xuan ${ }^{1}$, Toan Hoang Van ${ }^{1}$, Truong Vu${ }^{1}$ \\ ${ }^{1}$ Medicinal Supplies, Medical Physics Division, Hanoi Oncology Hospital, Hanoi, Vietnam \\ ${ }^{2}$ Radiotherapy Department, Hanoi Oncology Hospital, Hanoi, Vietnam \\ Email: *soaidq@bvubhn.vn, quangbv@bvubhn.vn, cuongbx@bvubhn.vn, hoangtoan1586@gmail.com, \\ vutruong29989@gmail.com
}

How to cite this paper: Dang Quoc, S., Bui Vinh, Q., Bui Xuan, C., Hoang Van, T. and $\mathrm{Vu}, \mathrm{T}$. (2020) Analyzing Planning Techniques for Whole Brain Radiotherapy. International Journal of Medical Physics, Clinical Engineering and Radiation Oncology, 9, 1-13. https://doi.org/10.4236/ijmpcero.2020.91001

Received: October 27, 2019

Accepted: December 8, 2019

Published: December 11, 2019

Copyright (C) 2020 by author(s) and Scientific Research Publishing Inc. This work is licensed under the Creative Commons Attribution International License (CC BY 4.0).

http://creativecommons.org/licenses/by/4.0/

\begin{abstract}
Aims. This study compares data between the Field in Field planning and Wedge planning techniques to figure out which technique has better dose coverage and distribution for PTV, and, if using FiF technique for whole brain treatment, how many beams will have better plan. Methods. 56 patients, who need to radiate whole brain with $30 \mathrm{~Gy} / 10$ fractions, have been selected for this study. Four plans have been made for each patient (FiF1-one subfield per field plan, FiF2-two subfields per field plan, FiF3-three subfields per field plan, and a Wedge plan). Results. The results of Field in Field plans including Compare dose distribution on the transverse CT slice, plan evaluation using DVH, number $M U$ of plan, $D_{\max } H I$, HTCI, $D_{\max P T V}, D_{\operatorname{mean} P T V}$. Volume of PTV with the dose over 105\% prescribed dose, dose of organ at risk, and Quality Assurance ( $Q A)$ plan, are better than those of Wedge plan. Conclusions. Plans using Field in Field technique has better coverage, is more homogeneous in dose distribution than plan using Wedge technique. When using Field in Field technique for whole brain radiotherapy, using three subfields per field has better result than two subfields per field and one subfield per field.
\end{abstract}

\section{Keywords}

Field in Field, Whole Brain Radiotherapy, HI, HTCI, Gamma Index

\section{Introduction}

The task of a medical physicist in the treatment planning process is to ensure that the targets receive a minimum of $95 \%$ and a maximum of $107 \%$ of the pre- 
scribed dose, and minimum doses, which affect organs at risk. The aim of this research is to figure out: 1) Whether using planning technique in whole brain treatment Field in Field or using Wedges planning technique is better for the patients and 2) When using Field in Field technique for whole brain treatment, how many subfields in field are better for patients [1]-[8].

The wedge, which is used in radiotherapy, is a wedge-shaped piece of lead or lead alloy. It is placed in the field to create the slope of the radiation intensity in the field. The thick end of the wedge is called the wedge heel; the thin end of the wedge is the wedge tip. The angle of the wedge is defined as the angle between the $50 \%$ isodose line and the line perpendicular to the field axis. The wedge angle is usually in the range from $10^{\circ}$ to $60^{\circ}$ [2].

There are three types of wedges currently in use: physics wedge, automatic wedge, and dynamic wedges. The physics wedge is the one removable by the LINAC operator. The automatic wedge is inserted and removed from the field by the remote control. The dynamic wedge is used to create a change in the intensity of the photon beam, which is similar to using the wedge by moving the accelerator's jaw while LINAC emits radiation. The wedge we used in this research is dynamic wedge [2]. Wedges are used for two main purposes: 1) To compensate the slope of the patient's body surface or density of tissue; 2) To reduce hotpot when using nonparallel field pair [2].

Field in Field planning technique is one of the most popular 3D treatments planning technique all over the world. This planning technique also has become more and more popular in Vietnam. Regarding research on Field in Field planning technique, Zoran Stefanovski and his colleague have published the article "Advantages of the technique with segmented fields for tangential breast irradiation [4], and G. Yanas and other researchers have researched on comparing the chest wall dose between Field in Field plan and the traditional 3D planning technique [2].

The Field in Field planning technique is used to create one or more subfield in the primary field. These subfields have the same parameters: Field size, gantry angle, collimator angle, and couch angle. The subfield differs from primary field in the MLC position, and the MU numbers that are shot in the subfield, are usually smaller than the MU number LINAC fires in the primary field. Therefore, in order to apply the Field in Field technique, required facilities must include an accelerator with MLC and a treatment planning software that has the Field in Field planning feature. The Field in Field planning technique is also used for two main purposes: 1) To reduce the dose at the hotpot and 2) To increase the dose at the coldpot shaped due to the slope on the patient's body surface and changes in tissue density in the patients' body by creating subfield with MLC that mask hotpot and open MLC at coldpot in the primary field or due to not using parallel pair. The use of subfield in the primary field therefore will help to decrease dose in hotpot and increase the dose for coldpot in the plan without the need to use wedges or bolus [2]. 


\section{Materials and Methods}

All patients of Hanoi Oncology Hospital receiving whole brain radiotherapy from February 2017 to September 2018 were selected for this study. There were totally 54 selected patients including 38 males and 16 females, age ranging from 18 years old to 75 year old. They were all diagnosed with lung cancer plus brain metastasis. All CT images of these patients required no scattering due to metal objects; the slice thickness is $5 \mathrm{~mm}$; all have contoured PTV, left eye, right eye, left len, and right len. Plus, all patients have been prescribed 30 Gy per ten fractions.

\subsection{Research Methodology}

First step: Each whole brain treatment patient is made four plans: One Field in Field plan that has one subfield in a main field (FiF1), one Field in Field plan that has two subfields in a main field (FiF2), one Field in Field plan that has three subfields in a main field (FiF3), and one plan using Wedge technique (W). After that the verification plan is made for every plan as shown in Figure 1.

Second step: Using Dose Volume Histograms (DVH) lines, 2D (two directions) dose distribution, Homogeneity Index (HI), Healthy Tissues Conformity Index (HTCI), dose parameter of PTV, and dose parameter of organ at risk to compare four plans.

Third step: Quality Assurance (QA) is made for all plans using the Delta 4 Scandidos equipment. In the software of the QA equipment we set 3\% Dose Difference (DD), $3 \mathrm{~mm}$ Distance to Agreement (DTA). The gamma index $\gamma\left(r_{m}\right)$ is calculated with the following formula:

$$
\begin{gathered}
\gamma\left(r_{m}\right)=\min \left\{\Gamma\left(r_{m}, r_{c}\right)\right\} \forall\left(r_{c}\right) \leq 1 \\
\left(r_{m}, r_{c}\right)=\sqrt{\frac{r^{2}\left(r_{m}, r_{c}\right)}{\Delta d_{m}^{2}}+\frac{\delta^{2}\left(r_{m}, r_{c}\right)}{\Delta D_{M}^{2}}} \\
r\left(r_{m}, r_{c}\right)=\left|r_{m}, r_{c}\right| \\
\delta\left(r_{m}, r_{c}\right)=D_{c}\left(r_{c}\right)-D_{m}\left(r_{m}\right)
\end{gathered}
$$

( $r_{m}$ is the measured dose distribution point; $r_{c}$ is the calculated dose distribution point, $\Delta d_{m}=3 \mathrm{~mm} ; \Delta D_{M}=3 \%, D\left(r_{c}\right)$ is the absolute dose at $r_{\dot{c}} D\left(r_{m}\right)$ is the absolute dose at $r_{m}$.)

The QA results of four plans of each patient then are compared with each other.

\subsection{Data Collection}

Statistics of PTV include: 3D maxdose of a plan $\left(D_{\max }\right)$, 3D maxdose of PTV $\left(\mathrm{D}_{\max P T V}\right)$, 3D mindose of PTV $\left(D_{\min P T V}\right)$, 3D meandose of PTV $\left(D_{\text {mean PTV }}\right), \mathrm{D}_{95 \%}$, $\mathrm{D}_{5 \%}, \mathrm{~V}_{95 \%}$, and volume of PTV $\left(V_{P T V}\right)$ as shown in Figure 2. The mean value and standard deviation of each parameter are plotted in the same graph.

Dose of organ at risk statistics include: Max dose of the left len $\left(D_{\max L L}\right)$, mean dose of the left len $\left(D_{\operatorname{mean} L L}\right)$, max dose of the left eye $\left(D_{\max L E}\right)$, mean dose of the 


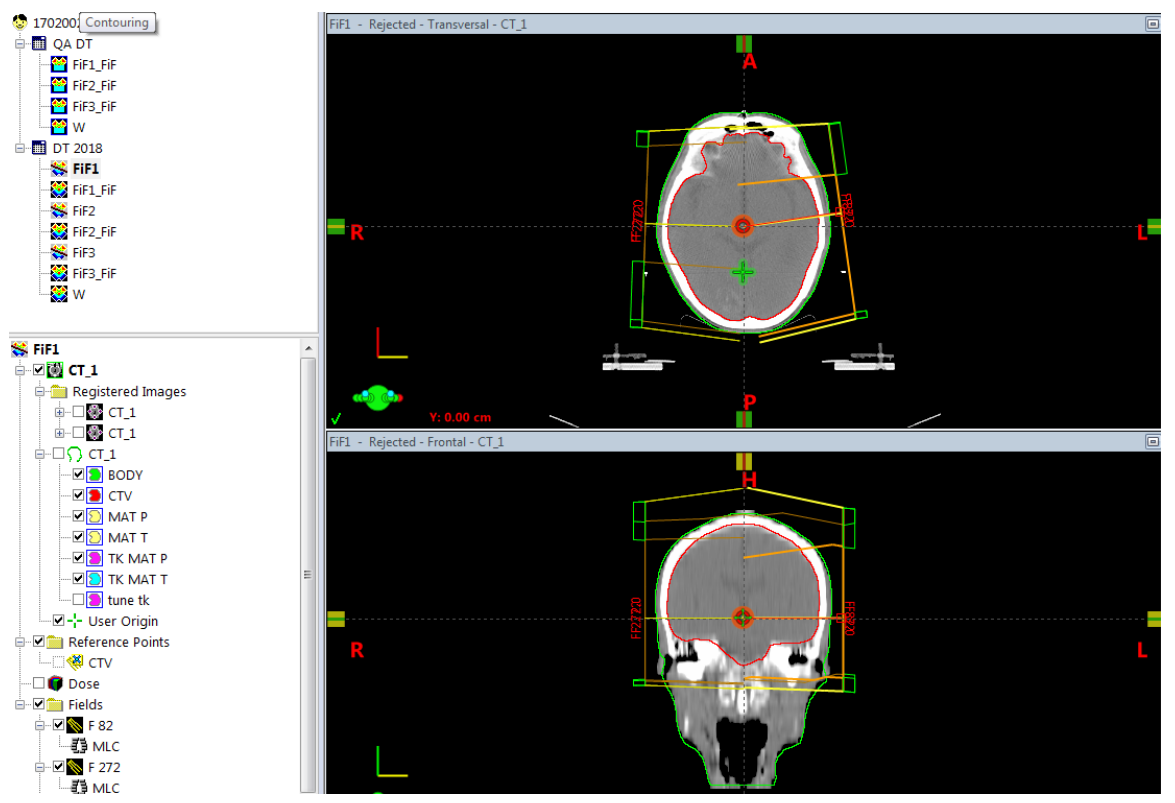

Figure 1. Each patient has four plans (FiF1, FiF2, FiF3, W) and four verification plans. It is taken from Eclipse 13.5.
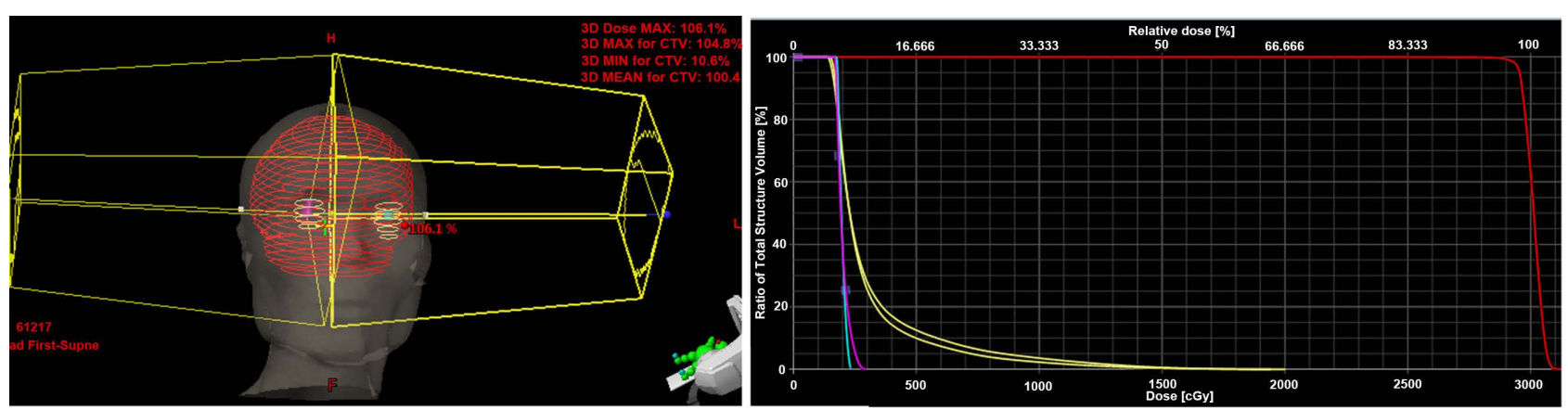

Figure 2. Collection of plan parameters and dose parameter for PTV. The left image is 3D view of plan, the right image is DVH of plan. It is taken from Eclipse 13.5.

left eye $\left(D_{\operatorname{mean} L E}\right)$, max dose of the right eye $\left(D_{\max R E}\right)$, and mean dose of the right eye $\left(D_{\text {meanRE }}\right)$ as shown in Figure 3 . The mean value and standard deviation of each parameter are plotted in the same graph.

$\mathrm{HI}$ is the parameter used to evaluate the homogeneity of dose distribution. The ideal value of HI is zero. Therefore, the smaller HI value of plan is, the more homogenous dose plan is (8).

$$
H I=100 \% \times \frac{D_{5 \%}-D_{95 \%}}{D_{p r e}}
$$

( $D_{\text {pre }}$ is the prescribed dose, $D_{5 \%}$ dose is corresponding to $5 \%$ of volume of PTV on the DVH $D_{95 \%}$ dose corresponding to 95 percent of volume of PTV on the DVH).

HTCI parameter characterizes dose coverage of PTV. The ideal value of HTCI is one. It means the closer to one HTCI is, the better coverage the plan has. HTCI is calculated with the following formula: 


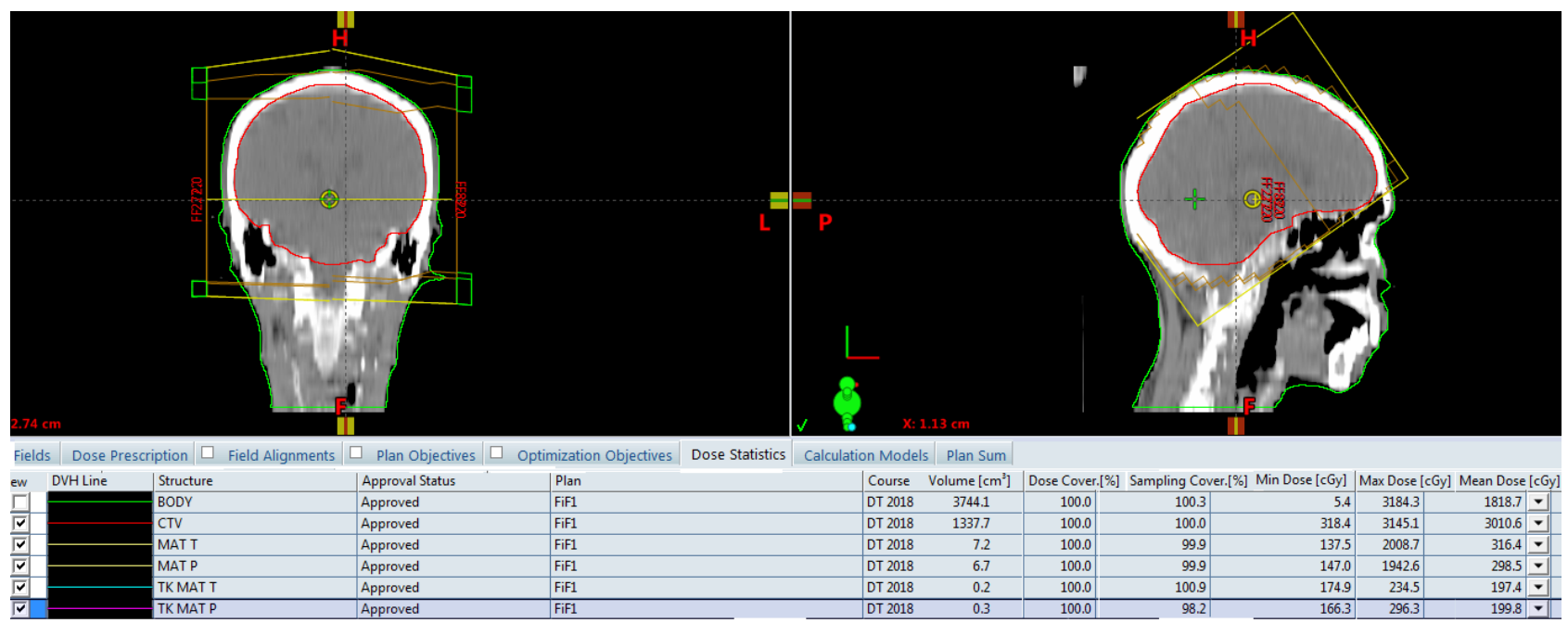

Figure 3. Collection parameters for organ at risk. It is taken from Eclipse 13.5.

$$
H T C I=\frac{V_{95 \%}}{V_{P T V}}
$$

( $V_{P T V}$ is volume of Planning Treatment Volume-PTV, $V_{95 \%}$ is volume of PTV which is covered with $95 \%$ of prescribed dose).

\section{Results}

a) Compare dose distribution on the transverse CT slice

By observing and comparing the dose distribution on each transverse CT image of four plans of the same patient, Figure 4 showed that $95 \%$ isodose line of plan using Field in Field technique covers PTV better than 95\% isodose line of the plan using Wedge technique.

\section{b) Plan evaluation using $D V H$}

When comparing DVH of four plans of the same patient, the authors realize that the volume of whole brain, that has the dose higher than the prescribed dose, decreases orderly W, FiF1, FiF2, FiF3; and the volume of whole brain, that has the dose lower than the prescribed dose, also decreases orderly W, FiF1, FiF2, and FiF3 as shown in Figure 5.

c) Number $M U$ of plan [9].

Figure 6 shows that the MU number per plan increases orderly FiF1, FiF2, FiF3, W. It means the radiation time of plan increases orderly FiF1, FiF2, FiF3, W.

\section{d) Results of plan $D_{\max }$}

According to Figure 7, the plan $D_{\max }$ of FiF1, FiF2, FiF3 are sequentially $0.65 \% ; 2.05 \% ; 3.04 \%$, less than the plan $D_{\max }$ recommended by ICRU 50 which is $107 \%$. Meanwhile the plan $D_{\max }$ of the Wedge plan is $0.98 \%$ higher than the plan $\mathrm{D}_{\max }$ recommended by ICRU 50. It means the Wedge plan $D_{\max }$ does not match the ICRU 50 recommendation, while the Field in Field plans well match the ICRU 50 recommendation (1). 


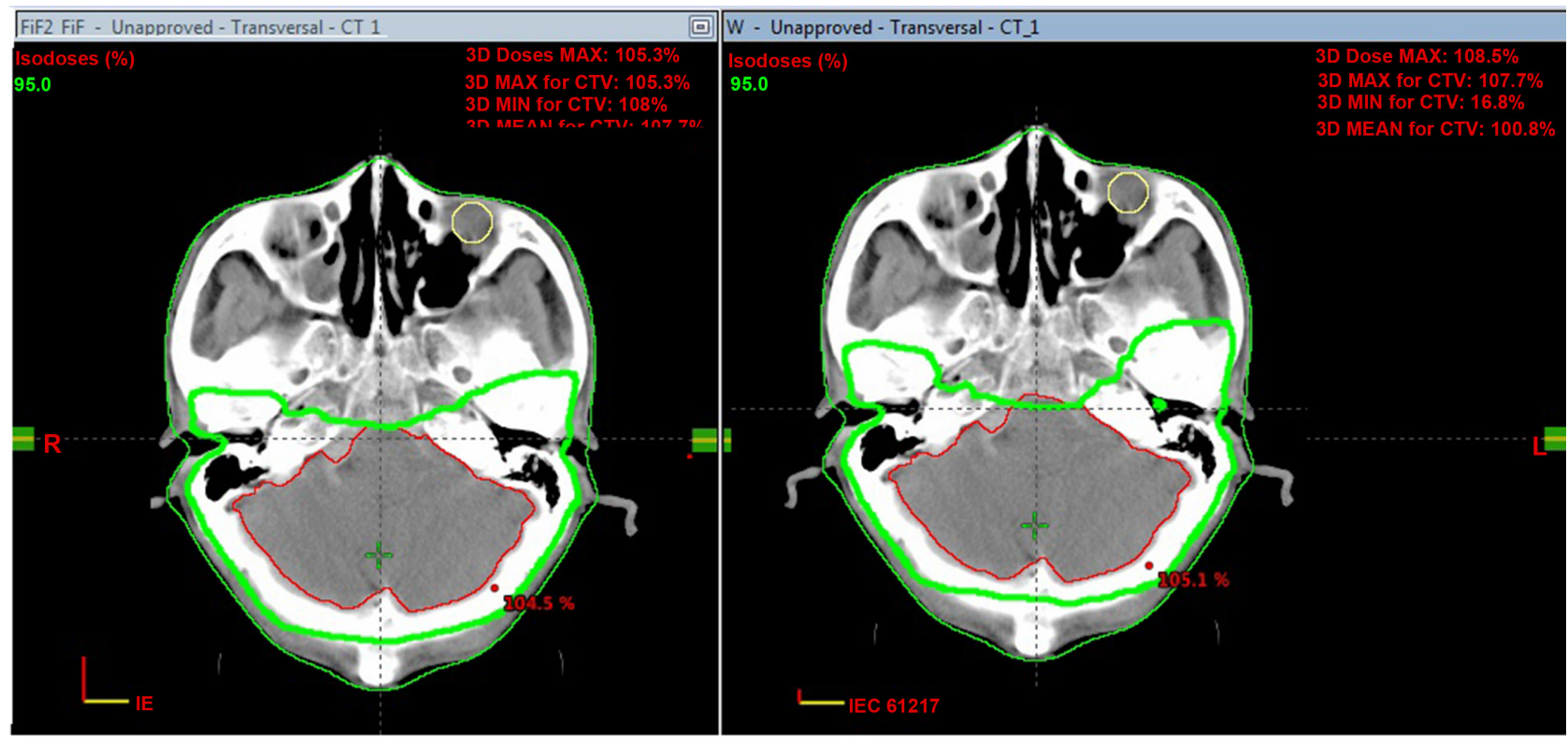

Figure 4. Compare dose distribution in the transverse slice of FiF1 plan (left image) with those of W plan (right image). It is taken from Eclipse 13.5.

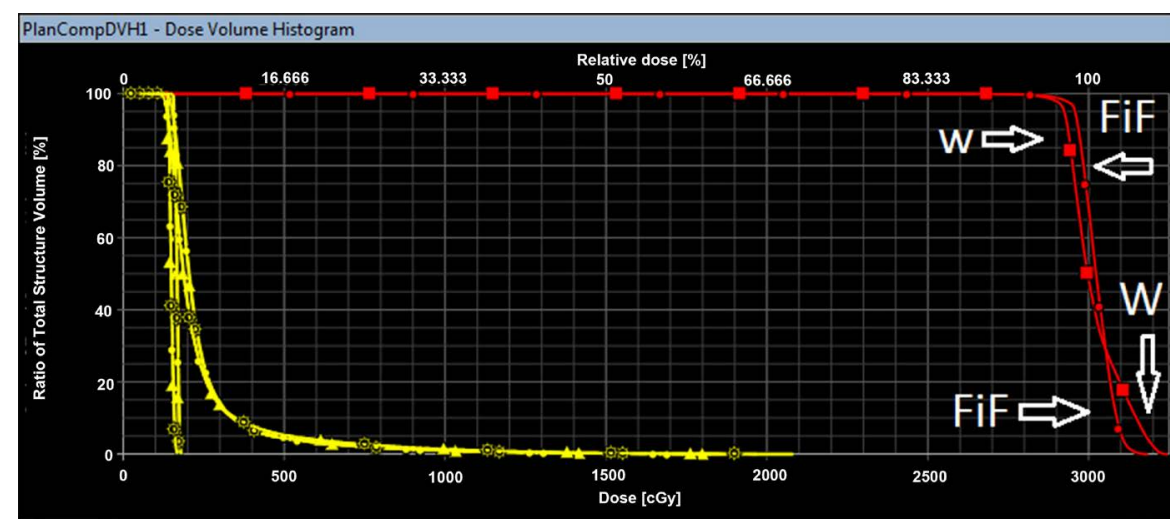

Figure 5. DVH of FiF1 plan (circle dot line) and DVH of W plan (square dot line). It is taken from Eclipse 13.5.

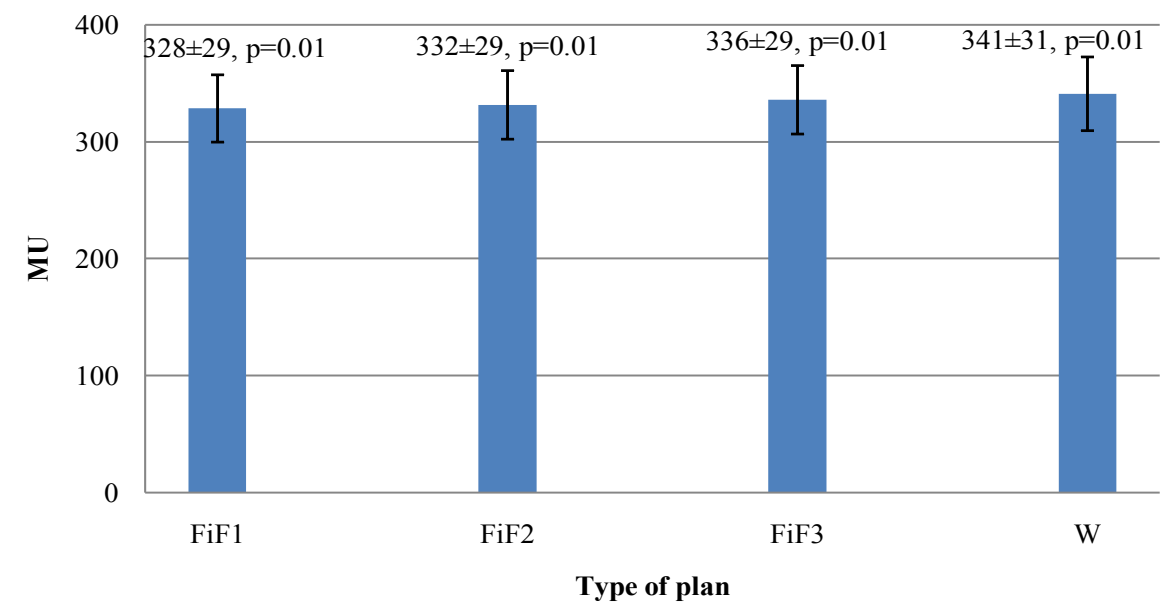

Figure 6. Average MU number per plan of four types of plan FiF1, FiF2, FiF3, W. 


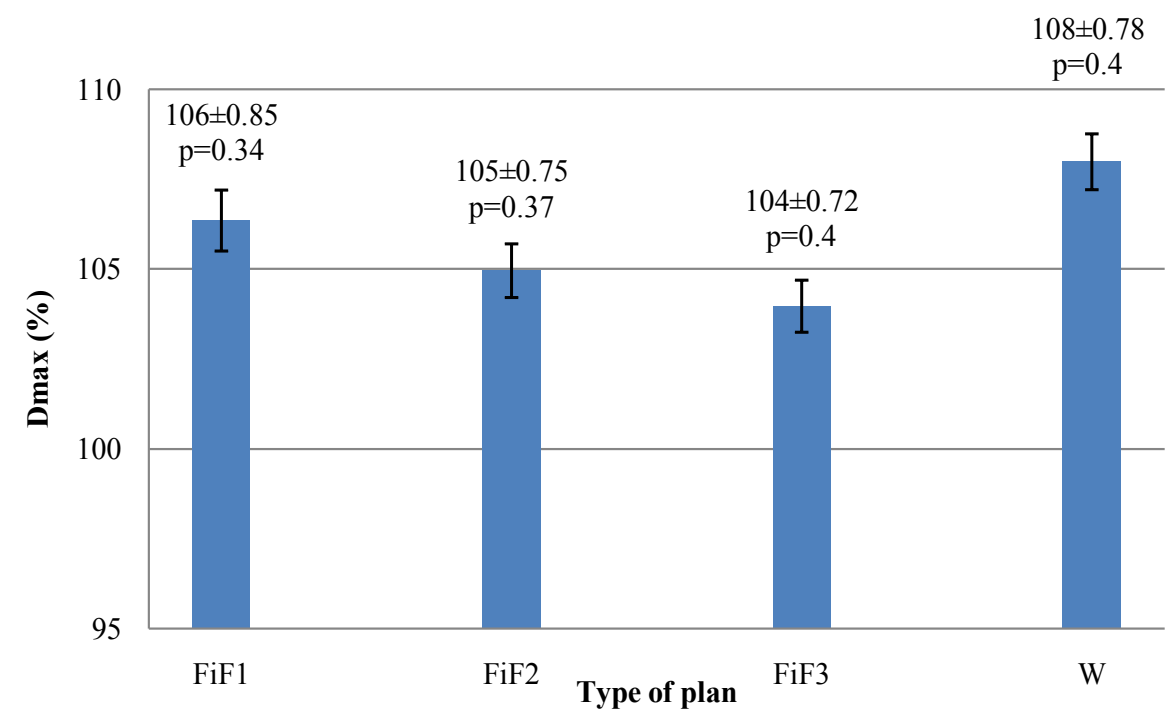

Figure 7. Average value of $D_{\max }$ per four types of plan FiF1, FiF2, FiF3, W.

\section{e) Homogeneity Index (HI)}

Figure 8 shows that the HI of the FiF3 plan is the closest to zero value, followed by the FiF2, FiF1, and W plans. According to the data collection part, the dose of PTV in the FiF3 plan is the most homogeneous one and then the homogeneity decreases in $\mathrm{FiF} 2, \mathrm{FiF} 1$, and $\mathrm{W}$ orderly.

\section{f) Healthy Tissues Conformity Index (HTCI)}

Figure 9 shows that the HTCIs of plans using Field in Field technique are similar to each other. The HTCI of the Wedge plan, however, is smaller than the HTCI of plans using Field in Field technique, and further from 1 (value One). As mentioned above, that means the distribution dose of the Field in Field plans cover PTV better than that of the Wedge plan

g) $D_{\max P T V}, D_{\text {mean } P T V}$

Figure 10 demonstrates that the mean dose and the max dose of PTV of the FiF3 plan are the closest to the prescribed dose, followed by the FiF2, FiF1, and then the $\mathrm{W}$ plan. That means the plans with three subfields in a main field has the best response to the treatment request. The second best response is the plan with two subfields in a main field, followed by the plan with one subfield in a main field; while the plan using the Wedge technique has the worst response to the treatment request.

h) Volume of PTV with the dose over $105 \%$ prescribed dose.

Figure 11 shows that the volume of PTV with the dose over 105\% prescribed dose of both the FiF3 plan and the FiF2 plan are the closest to zero value, far from that of the FiF1 plan which is $16 \mathrm{~cm}^{3}$; The biggest volume of PTV with the dose over $105 \%$ prescribed dose is $19.7 \mathrm{~cm}^{3}$, which belongs to the Wedge plan.

i) Dose of organ at risk

Regarding the doses of the organs at risk, Figure 12 shows that doses of the organs at risk in four types of plan are quite similar. 


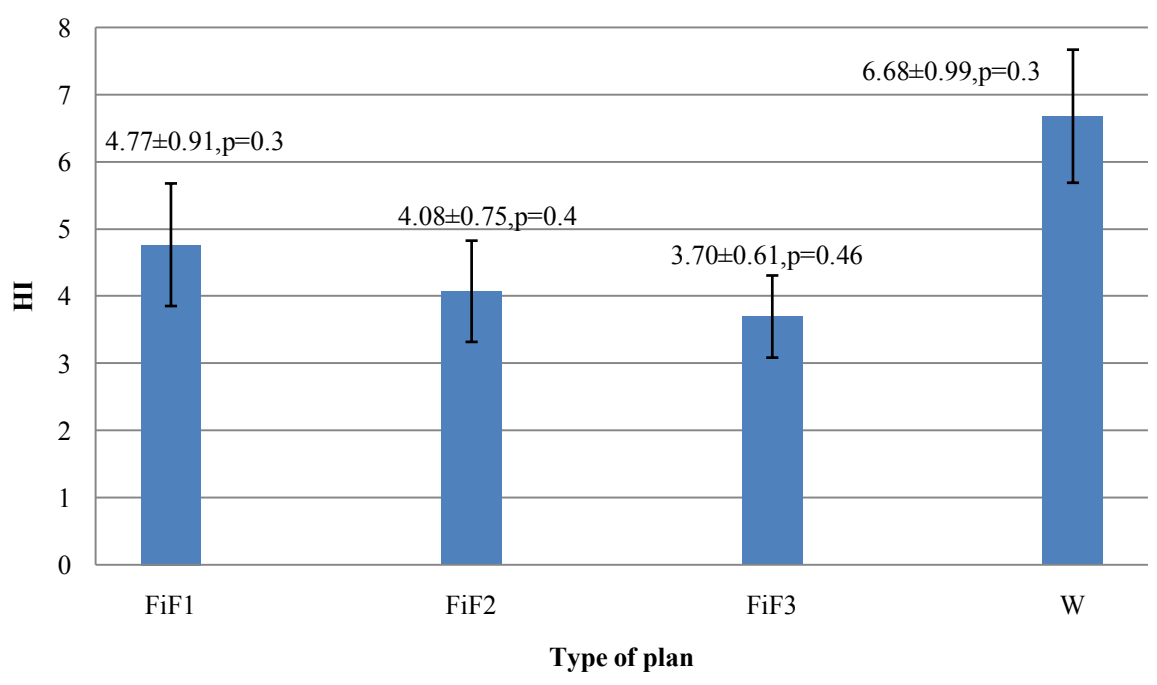

Figure 8. HI value of four types of plan FiF1, FiF2, FiF3, W.

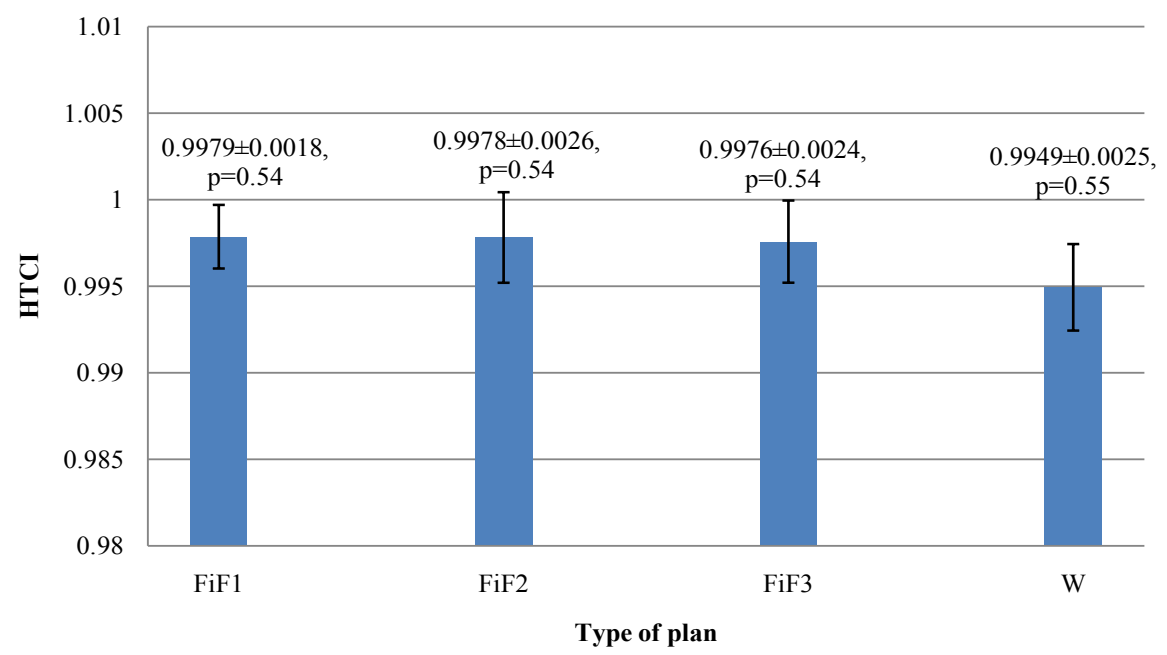

Figure 9. HTCI value of four types of plan FiF1, FiF2, FiF3, W.

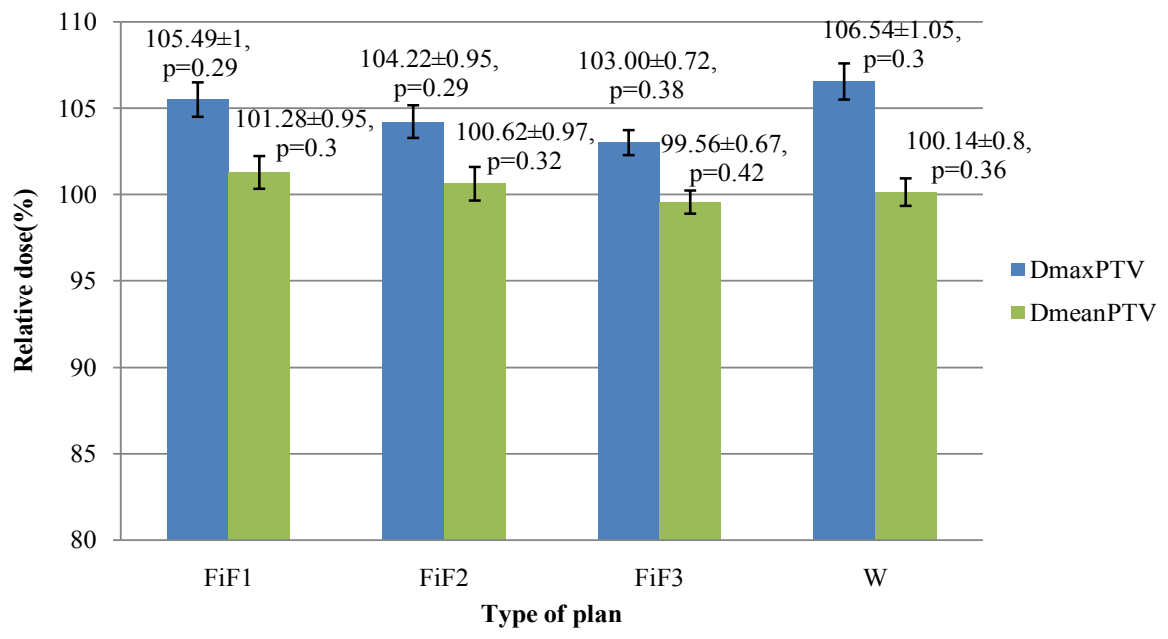

Figure 10. 3D max dose of PTV $\left(D_{\max P T V}\right)$, 3D mean dose of PTV $\left(D_{\text {mean } P T V}\right)$ of four types of plan FiF1, FiF2, FiF3, W. 


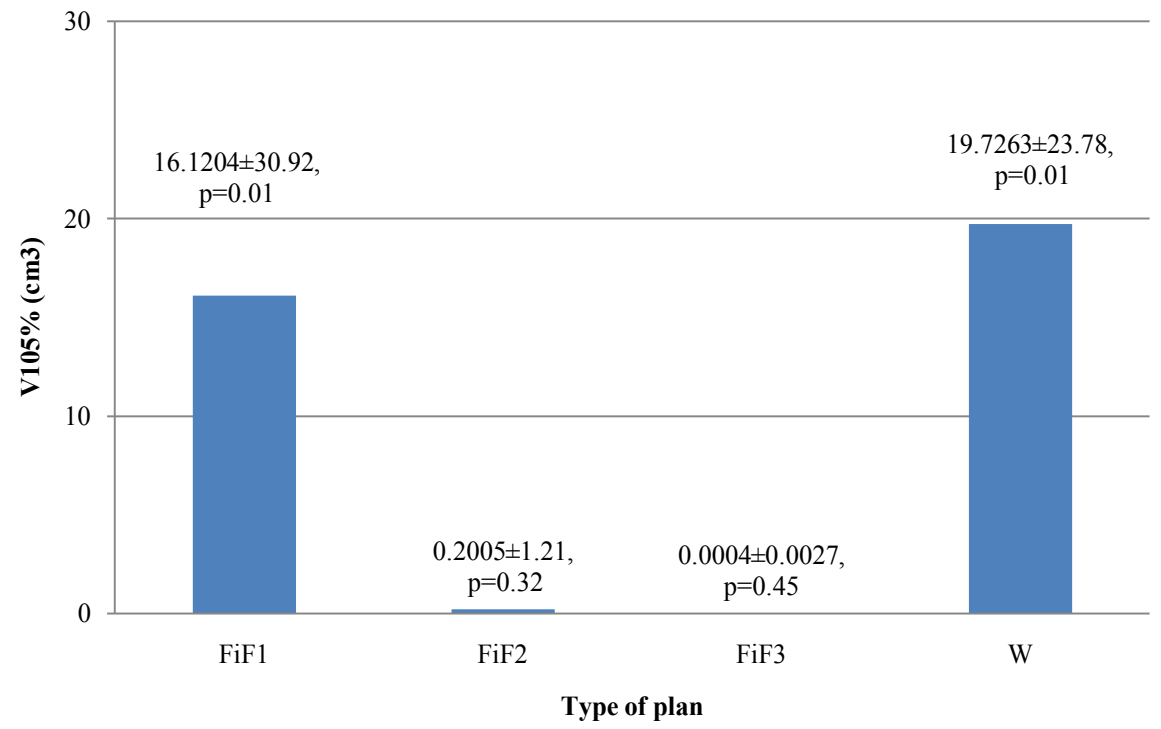

Figure 11. Volume of PTV with the dose over 105\% prescribed dose of four plan types FiF1, FiF2, FiF3, and W.

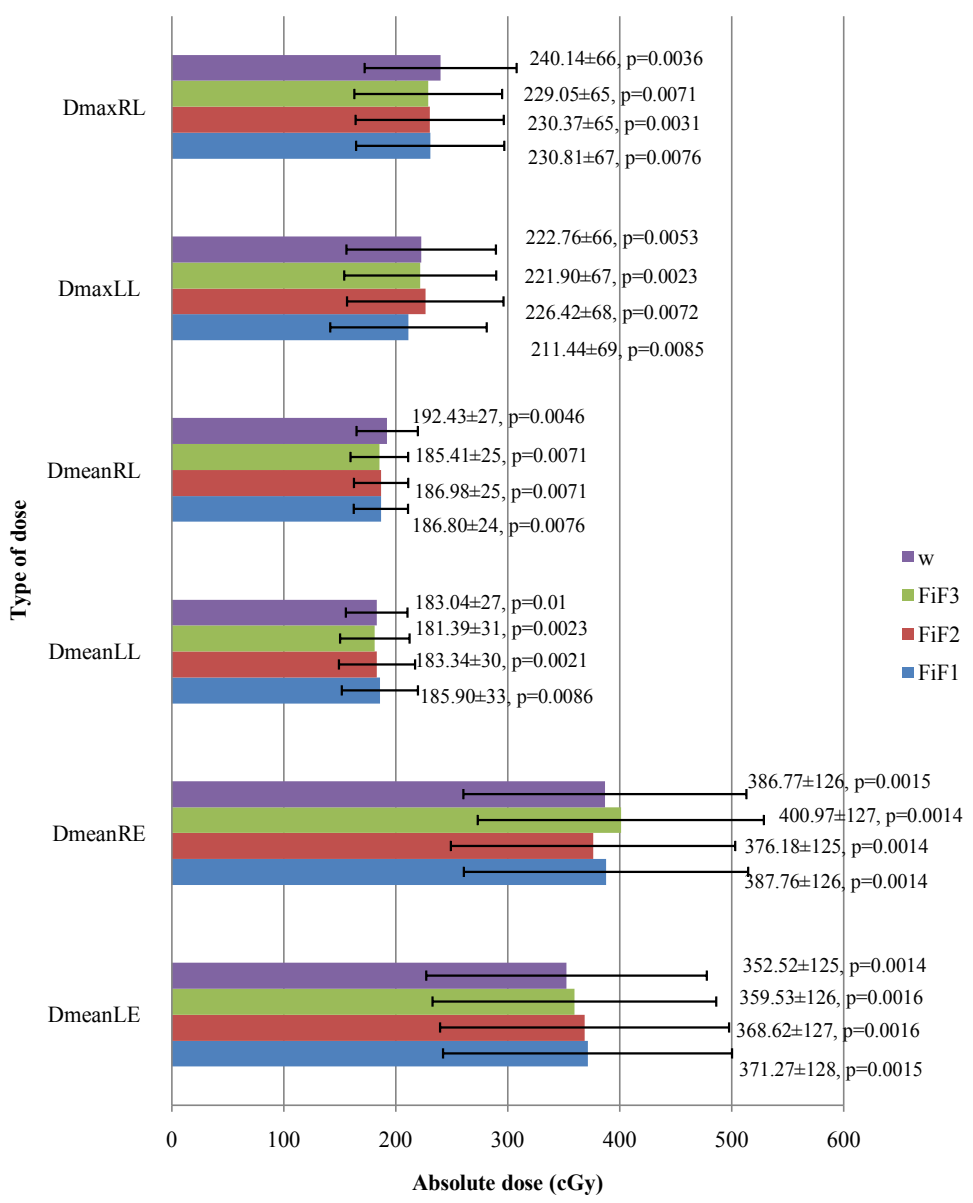

Figure 12. Absolute dose of organ at risk of four plan types (left len, left eye, right len, and right eye). It includes max dose of right len $\left(\mathrm{D}_{\max R L}\right)$; Max dose of left len $\left(\mathrm{D}_{\max L L}\right)$; Mean dose of right len $\left(\mathrm{D}_{\operatorname{mean} R L}\right)$; Mean dose of left len $\left(\mathrm{D}_{\text {mean } L L}\right)$; Mean dose of right eye $\left(\mathrm{D}_{\text {mean } R E}\right)$; Mean dose of left eye $\left(\mathrm{D}_{\text {mean } L E}\right)$. 


\section{j) The Quality Assurance ( $Q A)$}

Regarding QA plan result, the DDs, DTAs, Gamma Indexes of all plans are collected. The QA plan results of the Wedge plan are: average $\mathrm{DD}=35$; average $\mathrm{DTA}=93$; average of gamma index $=48$, then the values of the FiF plans are subtracted with those values of the Wedge plan. As presented in Figure 13, all subtracted values are positive ones with FiF3 has highest subtracted values. It means the FiF3 plan has the best QA plan results, followed by FiF2, and then FiF1. The plan using Wedge technique has the worst QA plan results.

\section{Discussion}

Since human's head has a globular shape, if the 3D whole brain radiotherapy does not use the Field in Field planning technique or the Wedge planning technique, there will be coldpot in the center of the head, and some hotpots in the skull bones. When using the Wedge planning technique, these hotpots can be eliminated, but the coldpot is still an unsolved issue. However, when using the Field in Field planning technique, both the coldpot and the hotpot are solved with the subfield. It is because the PTV of Field in Field plan is covered with the 95\% isodose line better than of the Wedge plan as we saw in the Results section part a) Compare dose distribution on the transverse CT slice and b) Plan evaluation using $D V H$. In addition, the Field in Field plans have less hotpots than the Wedge plans does, as proved in d) Results of plan $\left.D_{\max }, \mathrm{g}\right) D_{\max P T V}, D_{\operatorname{mean} P T V}$ results and h) Volume of PTV with the dose over $105 \%$ prescribed dose. According to those results, the dose distribution of the Field in Field plans is more homogeneous, covers better than the one of the Wedge plan. It can be seen in b) Plan evaluation using $D V H$, e) $H I$ results, f) $H T C I$ results, and j) The QA results of plan.

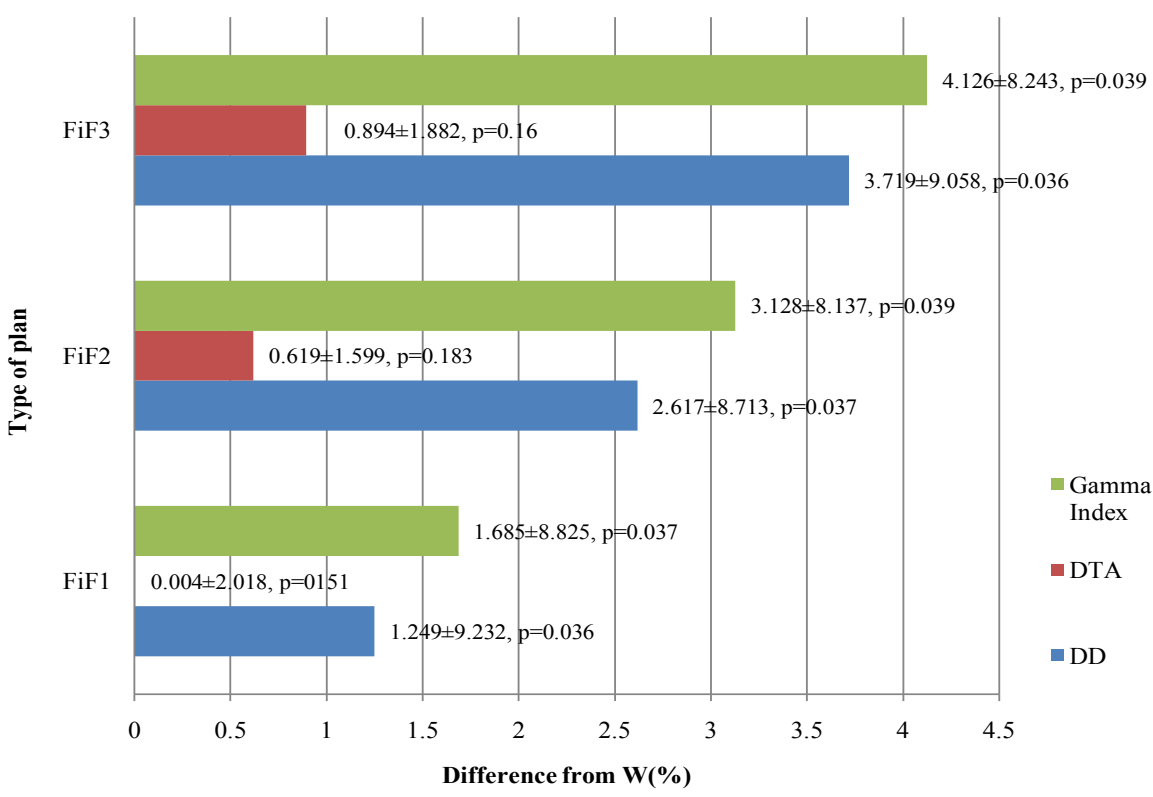

Figure 13. QA plan values of FiF1, FiF2, FiF3 plans after being subtracted the QA value of plan using Wedge. 
When a radiation beam passes through the Wedge, it becomes harder because it has lost its' low energy part. Therefore, if the target needs to get the same dose as with the beam in open field, the LINAC must shoot more MUs. This is the reason the average MU number of the Wedge plan is higher than the one of the Field and Field plan. Plus, when the Field in Field plan has one more subfield, it also increases the MU number, so the average MU number of the FiF3 plan is higher than the FiF1 and FiF2 plans.

Regarding the organs at risk, even though they are outside of the beam field size, they may be affected by the radiation leakage from the head of LINAC and by the scattering radiation. That explains the similar doses of the organs at risk of the Field in Field plans and the Wedge plan.

\section{Conclusion}

The collected results and above data analysis prove that in the whole brain treatment, using the Field in Field planning technique ensures more homogenous and better covered dose distribution than using the Wedge planning technique. Therefore, it is recommended for medical physicists to choose the Field in Field planning technique over the Wedge planning technique for whole brain treatment. Plus, when using Field in Field technique planning for the whole brain treatment, using three subfields in a main field is better for patients than using two subfields or one subfield in a main field.

\section{Acknowledgements}

The research facilities and manuscript submission are approved and sponsored by Hanoi Oncology Hospital. The authors would like to thank Dr. Nguyen Thanh Hang from International Cooperation and Scientific Research Unit of Hanoi Oncology Hospital, for editing the manuscript and providing insight and expertise that greatly assisted the study.

\section{Conflicts of Interest}

The authors declare no conflicts of interest regarding the publication of this paper.

\section{References}

[1] Hinckley, D. (1994) Prescribing, Recording, and Reporting Photon Beam Therapy. ICRU 50, Medical Physics.

[2] Parker, W. and Horacio, P. (2003) Review of Radiation Oncology Physics: A Handbook for Teachers and Students, Chapter 7. The IAEA Publication, 179-224.

[3] Stefanovski, Z., Smichkoska, S., Petrova, D. and Lazarova, E. (2013) Advantages of the Technique with Segmented Fields for Tangential Breast Irradiation. UDC: 618.19-006.6-085.849.1.

[4] Yanas, G., Yavas, C. and Avar, H. (2012) Dosimetric Comparison of Whole Breast Radiotherapy Using Field in Field and Conformal Radiotherapy Techniques in Early stage Breast Cancer. International Journal of Radiation Research, 10, 131-138. 
[5] Khan, F.M. (2003) Physics of Radiation Therapy. 3rd Edition, Includes Bibliogrpahical References and Index.

[6] Khan, F.M., Gibbons, J.P. and Sperduto, P.W. (2006) Treatment Planning in Radiation Oncology. 4th Edition, Lippincott Williams \& Wilkins.

[7] Li, H., Dong, L., Zhang, L., Yang, J.N., Gillin, M.T. and Zhu, X.R. (2011) Toward a Better Understanding of the Gamma Index: Investigation of Parameters with Surface-Based Distance Method. Medical Physics, 38, 6730-6741. https://doi.org/10.1118/1.3659707

[8] Kataria, T., Sharma, K., Subramani, V., Karrthick, K.P. and Bisht, S.S. (2012) Homogeneity Index: An Objective Tool for Assessment of Conformal Radiation Treatments. Journal of Medical Physics, 37, 207-213.

https://doi.org/10.4103/0971-6203.103606

[9] Ministry of Science and Technology, Vietnam. (2017) Quy chuẩn kỹ thuật Việt nam cho máy gia tốc dùng trong y (Vietnamese Technical Regulation for Accelerators Used in Medicine).

https://luatvietnam.vn/y-te/quy-chuan-viet-nam-qcvn-13-2017-bkhcn-bo-khoa-hoc -va-cong-nghe-156309-d3.html 


\section{List of Abbreviations}

\begin{tabular}{|c|c|}
\hline $2 \mathrm{D}$ & Two directions \\
\hline $\mathrm{CT}$ & Computed Tomography \\
\hline$D_{5 \%}$ & Dose corresponding to $5 \%$ of volume of PTV on the DVH \\
\hline$D_{95 \%}$ & Dose corresponding to 95 percent of volume of PTV on the DVH \\
\hline$D D$ & Dose Difference \\
\hline$D_{\max }$ & 3D max dose of a plan \\
\hline$D_{\max L E}$ & Max dose of the left eye \\
\hline$D_{\max L L}$ & Max dose of the left len \\
\hline $\mathrm{D}_{\operatorname{maxPTV}}$ & 3D max dose of PTV \\
\hline $\mathrm{D}_{\operatorname{maxRE}}$ & Max dose of the right eye \\
\hline$D_{\operatorname{mean} L E}$ & Mean dose of the left eye \\
\hline$D_{\text {mean } L L}$ & Mean dose of the left len \\
\hline$D_{\text {mean } P T V}$ & 3D mean dose of PTV \\
\hline $\mathrm{D}_{\text {meanRE }}$ & Mean dose of the right eye \\
\hline$D_{\min P T V}$ & 3D min dose of PTV \\
\hline DTA & Distance to Agreement \\
\hline DVH & Dose Volume Histograms \\
\hline $\mathrm{FiF}$ & Field in Field \\
\hline FiF1 & Field in Field plan has one subfield in a main field \\
\hline FiF2 & Field in Field plan has two subfields in a main field \\
\hline FiF3 & Field in Field plan has three subfields in a main field \\
\hline HI & Homogeneity Index \\
\hline HTCI & Healthy Tissues Conformity Index \\
\hline LINAC & Linear Accelerator \\
\hline MU & Machine Unit \\
\hline PTV & Planning Target Volume \\
\hline QA & Quality Assurance \\
\hline $\mathrm{V}_{95 \%}$ & Volume of PTV corresponding to $95 \%$ of prescribed dose on the DVH \\
\hline $\mathrm{V}_{5 \%}$ & Volume of PTV corresponding to $5 \%$ of prescribed dose on the DVH \\
\hline $\mathrm{V}_{\mathrm{PTV}}$ & Volume of PTV \\
\hline $\mathrm{W}$ & Wedge \\
\hline
\end{tabular}

